\title{
A CLASSIFICATION SCHEME FOR NONOSCILLATORY SOLUTIONS OF A HIGHER ORDER NEUTRAL NONLINEAR DIFFERENCE EQUATION
}

\author{
WAN-TONG LI*, SUI SUN CHENG and GUANG ZHANG
}

(Received 27 July 1998; revised 21 April 1999)

Communicated by E. N. Dancer

\begin{abstract}
Nonoscillatory solutions of a nonlinear neutral type higher order difference equations are classified by means of their asymptotic behaviors. Existence criteria are then provided for justification of such classifications.
\end{abstract}

1991 Mathematics subject classification (Amer. Math. Soc.): primary 39A10.

Keywords and phrases: Nonlinear neutral difference equation, nonoscillatory solution, superlinear function, sublinear function, existence criteria.

\section{Introduction}

Classifications schemes for nonoscillatory solutions of nonlinear difference equations are important since further investigations of some of the qualitative behaviors of nonoscillatory solutions can then be reduced to only a number of cases. In this paper, we are concerned with a class of nonlinear neutral difference equations of the form

$$
\Delta^{m}\left(x_{n}+p x_{n-\tau}\right)+f\left(n, x_{n-\delta}\right)=0, \quad n=0,1,2, \ldots,
$$

where $m, \tau$ and $\delta$ are integers such that $m \geq 2, \tau>0, \delta \geq 0$, and $p$ is a nonnegative real number different from 1 . The function $f:\{0,1, \ldots\} \times \mathbb{R} \rightarrow \mathbb{R}$ is continuous in the second variable and $x f(n, x)>0$ for $x \neq 0$ and all $n=0,1,2, \ldots$ In some cases, we will also assume that $f$ is superlinear or sublinear. More precisely, $f$ is said

* Supported by the Science Foundation of Gansu University of Technology.

(C) 1999 Australian Mathematical Society 0263-61 15/99 \$A2.00+0.00 
to be superlinear if

$$
\frac{f(n, x)}{x} \geq \frac{f(n, y)}{y}, \quad \text { for } x \geq y>0, \text { or } x \leq y<0,
$$

and sublinear if

$$
\frac{f(n, x)}{x} \leq \frac{f(n, y)}{y}, \quad \text { for } x \geq y>0 \text {, or } x \leq y<0 .
$$

Note that if $f$ is superlinear, then when $0<a \leq x \leq b$, we have

$$
f(n, a) \leq f(n, x) \leq f(n, b), \quad n=0,1,2, \ldots,
$$

and if $f$ is sublinear, then when $0<a \leq x \leq b$, we have

$$
\frac{a}{b} f(n, a) \leq f(n, x) \leq \frac{b}{a} f(n, b), \quad n=0,1, \ldots .
$$

Similar statements can be made when $a \leq x \leq b<0$.

Since (1) can be written as a recurrence relation

$$
x_{n+m}=F\left(x_{n-\delta}, x_{n-\tau}, x_{n}, x_{n+1}, \ldots, x_{n+m-1}\right),
$$

it is clear that given $x_{i}$ for $-\max \{\tau, \delta\} \leq i \leq 1$, we can successively calculate $x_{2}, x_{3}, \ldots$ in a unique manner. Such a sequence $\left\{x_{n}\right\}$ will be called a solution of (1). We will be concerned with the nonoscillatory, that is eventually positive or eventually negative solutions of (1). In particular, we will provide a classification scheme for these solutions. This scheme is then justified by existence criteria.

Nonlinear difference equations have been studied by a number of authors. In particular, Grace and Lalli in [4] studied the equation

$$
\Delta^{2}\left(x_{n}+p x_{n-\tau}\right)+q_{n} f\left(x_{n-\delta}\right)=0
$$

and obtained a number of oscillation criteria for its solutions. Li and Cheng in [10] studied the equation

$$
\Delta\left(r_{n} \Delta\left(x_{n}-p_{n} x_{n-\tau}\right)\right)+f\left(n, x_{n-\delta}\right)=0
$$

and obtained classification schemes for its nonoscillatory solutions. Fourth order equations of the form

$$
\Delta^{2}\left(r_{n+1} \Delta^{2} x_{n}\right)+f\left(n+2, y_{\tau(n)}\right)=0
$$

have been studied by a number of people (see [12-14]) and several results are related to classifications of nonoscillatory solutions. Other related results can be found in [1-5, 7-16].

The convention that an empty sum is zero will be adopted in the sequel. 


\section{Preparatory lemmas}

We first establish or quote several preparatory results which will be useful later. Let $\left\{x_{n}\right\}$ be a real sequence defined for $n \geq a-\tau$, the sequence $\left\{z_{n}\right\}$ defined by

$$
z_{n}=x_{n}+p x_{n-\tau}, \quad n=a, a+1, \ldots,
$$

will be called its associated sequence (relative to the constant $p$ and the integer $\tau$ ). Two relations between a nonoscillatory solution and its associated sequence will be needed.

Lemma 1. Assume that $p \geq 0$ and $p \neq 1$. Assume further that the sequence $\left\{x_{n} / n^{\alpha}\right\}$ is bounded and eventually positive (or eventually negative), where $\alpha$ is a nonnegative integer. If $\left\{z_{n}\right\}$ is the associated sequence of $\left\{x_{n}\right\}$ and

$$
\lim _{n \rightarrow \infty} \frac{z_{n}}{n^{\alpha}}=b,
$$

then

$$
\lim _{n \rightarrow \infty} \frac{x_{n}}{n^{\alpha}}=\frac{b}{1+p} .
$$

PROOF. Without loss of generality, we may assume that $x_{n}>0$ for $n \geq 0$. Let

$$
Q=\limsup _{n \rightarrow \infty} \frac{x_{n}}{n^{\alpha}} \quad \text { and } \quad q=\liminf _{n \rightarrow \infty} \frac{x_{n}}{n^{\alpha}} .
$$

Then there exist subsequences $\{n(k)\}$ and $\{j(k)\}$ of the nonnegative integers such that

$$
\lim _{k \rightarrow \infty} \frac{x_{n(k)}}{n^{\alpha}(k)}=Q \quad \text { and } \quad \lim _{k \rightarrow \infty} \frac{x_{j(k)}}{j^{\alpha}(k)}=q .
$$

If $p \in[0,1)$, then

$$
b=\lim _{k \rightarrow \infty} \frac{z_{n(k)}}{n^{\alpha}(k)}=\lim _{k \rightarrow \infty} \frac{x_{n(k)}+p x_{n(k)-\tau}}{n^{\alpha}(k)} \geq Q+p q
$$

and

$$
b=\lim _{k \rightarrow \infty} \frac{z_{j(k)}}{j^{\alpha}(k)}=\lim _{k \rightarrow \infty} \frac{x_{j(k)}+p x_{j(k)-\tau}}{j^{\alpha}(k)} \leq q+p Q .
$$

Hence $q+p Q \geq Q+p q$, or $q \geq Q$. Since $q \leq Q$ by definition, we see that $q=Q$.

Similarly, if $p>1$, we have

$$
b=\lim _{k \rightarrow \infty} \frac{z_{n(k)+\tau}}{(n(k)+\tau)^{\alpha}} \geq q+p Q
$$


and

$$
b=\lim _{k \rightarrow \infty} \frac{z_{j(k)+\tau}}{(j(k)+\tau)^{\alpha}} \leq Q+p q .
$$

Thus $Q+p q \geq q+p Q$, or $q \geq Q$. Again, we have $q=Q$.

Thus,

$$
b=\lim _{n \rightarrow \infty} \frac{z_{n}}{n^{\alpha}}=\lim _{n \rightarrow \infty} \frac{x_{n}+p x_{n-\tau}}{n^{\alpha}}=q+p q .
$$

This shows that

$$
\lim _{n \rightarrow \infty} \frac{x_{n}}{n^{\alpha}}=q=\frac{b}{1+p}
$$

The proof is complete.

Given a real function $u(t)$ whose derived function $u^{(r)}(t)$ is sign regular, the intermediate derived functions will also satisfy certain sign conditions. Such results are well known in the theory of ordinary differential equations (see Kiguradze and Chanturia [6]) and their discrete analogs have been reported by a number of authors $[13,16]$. Several of these sign regularity conditions are listed here without proofs.

LEMMA 2 (Zhou and Yan [16]). Let $\left\{x_{k}\right\}$ be a real bounded sequence of fixed sign. Suppose $x_{k} \Delta^{t} x_{k} \leq 0\left(x_{k} \Delta^{t} x_{k} \geq 0\right)$ for some odd integer $t>1$ and all large $k$. Then $(-1)^{j} x_{k} \Delta^{j} x_{k} \geq 0$ (respectively $(-1)^{j} x_{k} \Delta^{j} x_{k} \leq 0$ ) for $1 \leq j \leq t$ and all large $k$.

LEMMA 3 (Zafer and Dahiya [13]). Let $N$ be a positive integer. Let $\left\{y_{n}\right\}_{n=0}^{\infty}$ be a real sequence such that $\left\{y_{n}\right\}$ and $\left\{\Delta^{N} y_{n}\right\}$ are of constant sign. Suppose further that $\left\{\Delta^{N} y_{n}\right\}$ does not vanish identically for all large $n$ and that $y_{n} \Delta^{N} y_{n} \leq 0$ for $n \geq 0$. Then

(i) for each $j \in\{1,2, \ldots, N-1\}$, the sequence $\left\{\Delta^{j} y_{n}\right\}$ is of constant sign for all large $n$; and

(ii) there is an integer $k \in\{0,1,2, \ldots, N-1\}$ such that $(-1)^{N-k-1}=1$ and for each $j \in\{0,1, \ldots, k\}, y_{n} \Delta^{j} y_{n}>0$ for all large $n$, and for each $j \in\{k+1, \ldots, N-$ 1\}, $(-1)^{j-k} y_{n} \Delta^{j} y_{n}>0$ for all large $n$.

LEMMA 4 (Zafer and Dahiya [13]). Let $N$ be a positive integer. Let $\left\{y_{n}\right\}_{n=0}^{\infty}$ be a real sequence such that for each $j \in\{0,1, \ldots, N-1\},\left\{\Delta^{j} y_{n}\right\}$ is of constant sign for all large $n$. Suppose further that $y_{n} \Delta^{N} y_{n} \geq 0$ for all large $n$. Then either

(i) for each $j \in\{1,2, \ldots, N\}, y_{n} \Delta^{j} y_{n} \geq 0$ for all large $n$; or 
(ii) there is an integer $k \in\{0,1, \ldots, N-2\}$ such that $(-1)^{N-k}=1$, and for each $j \in\{1, \ldots, k\}, y_{n} \Delta_{n}^{j} y>0$ for all large $n$, and for each $j \in\{k+1, \ldots, N-2\}$, $(-1)^{j-k} y_{n} \Delta_{n}^{j} y_{n}>0$ for all large $n$.

We need the following result in our subsequent development. Let $B$ be the linear space of all real sequences $x=\left\{x_{n}\right\}_{n=N}^{\infty}$ endowed with the usual operations and the supremum norm

$$
\|x\|=\sup _{k \geq N} \frac{\left|x_{k}\right|}{h_{k}},
$$

where $\left\{h_{k}\right\}_{k=N}^{\infty}$ is a positive sequence with a uniform positive lower bound. Then $B$ is a Banach space. A set $\Omega$ of sequences in $B$ under the above norm is said to be uniformly Cauchy if for every $\epsilon>0$, there exists an integer $M$ such that whenever $i, j>M$, we have $\left|\left(x_{i} / h_{i}\right)-\left(x_{j} / h_{j}\right)\right|<\epsilon$ for all $x=\left\{x_{k}\right\} \in \Omega$. The following discrete Schauder type fixed point theorem was obtained by Cheng and Patula [2].

LEMMA 5. Let $\Omega$ be a closed, bounded and convex subset of the Banach space $B$. Suppose $T$ is a continuous mapping such that $T(\Omega)$ is contained in $\Omega$, and suppose the $T(\Omega)$ is uniformly Cauchy. Then $T$ has a fixed point in $\Omega$.

The following well known theorem of Stolz is a discrete analog of l'Hopital's rule (see [1, Theorem 1.7.7 and Corollary 1.7.8]).

LEMMA 6 (Stolz's theorem). Let $\left\{u_{k}\right\}$ and $\left\{v_{k}\right\}$ be two real sequences such that $v_{k}>0$ and $\Delta v_{k}>0$ for all large $k$. If $\lim _{k \rightarrow \infty} v_{k}=\infty$ and $\lim _{k \rightarrow \infty} \Delta u_{k} / \Delta v_{k}=c$, where $c$ may be infinite, then $\lim _{k \rightarrow \infty} u_{k} / v_{k}=c$.

\section{Classification scheme}

We will propose a classification scheme for the nonoscillatory solutions of (1). For this purpose it is convenient to denote the set of all eventually positive solutions of (1) by $S^{+}$and also make use of the following notations:

$$
\begin{aligned}
E_{j}(\infty, *) & =\left\{\left\{x_{n}\right\} \in S^{+} \mid \lim _{n \rightarrow \infty} \frac{x_{n}}{n^{2 j-2}}=\infty, \lim _{n \rightarrow \infty} \frac{x_{n}}{n^{2 j-1}} \in(0, \infty)\right\} \\
& =\left\{\left\{x_{n}\right\} \in S^{+} \mid \lim _{n \rightarrow \infty} \frac{x_{n}}{n^{2 j-1}} \in(0, \infty)\right\}, \\
E_{j}(\infty, 0) & =\left\{\left\{x_{n}\right\} \in S^{+} \mid \lim _{n \rightarrow \infty} \frac{x_{n}}{n^{2 j-2}}=\infty, \lim _{n \rightarrow \infty} \frac{x_{n}}{n^{2 j-1}}=0\right\}, \\
E_{j}(*, 0) & =\left\{\left\{x_{n}\right\} \in S^{+} \mid \lim _{n \rightarrow \infty} \frac{x_{n}}{n^{2 j-2}} \in(0, \infty), \lim _{n \rightarrow \infty} \frac{x_{n}}{n^{2 j-1}}=0\right\} \\
& =\left\{\left\{x_{n}\right\} \in S^{+} \mid \lim _{n \rightarrow \infty} \frac{x_{n}}{n^{2 j-2}} \in(0, \infty)\right\},
\end{aligned}
$$




$$
\begin{aligned}
O_{j}(\infty, *) & =\left\{\left\{x_{n}\right\} \in S^{+} \mid \lim _{n \rightarrow \infty} \frac{x_{n}}{n^{2 j-1}}=\infty, \lim _{n \rightarrow \infty} \frac{x_{n}}{n^{2 j}} \in(0, \infty)\right\} \\
& =\left\{\left\{x_{n}\right\} \in S^{+} \mid \lim _{n \rightarrow \infty} \frac{x_{n}}{n^{2 j}} \in(0, \infty)\right\}, \\
O_{j}(\infty, 0) & =\left\{\left\{x_{n}\right\} \in S^{+} \mid \lim _{n \rightarrow \infty} \frac{x_{n}}{n^{2 j-1}}=\infty, \lim _{n \rightarrow \infty} \frac{x_{n}}{n^{2 j}}=0\right\}, \\
O_{j}(*, 0) & =\left\{\left\{x_{n}\right\} \in S^{+} \mid \lim _{n \rightarrow \infty} \frac{x_{n}}{n^{2 j-1}} \in(0, \infty), \lim _{n \rightarrow \infty} \frac{x_{n}}{n^{2 j}}=0\right\} \\
& =\left\{\left\{x_{n}\right\} \in S^{+} \mid \lim _{n \rightarrow \infty} \frac{x_{n}}{n^{2 j-1}} \in(0, \infty)\right\},
\end{aligned}
$$

where $j$ is some integer to be specified. Similar notations can also be introduced for eventually negative solutions of (1).

THEOREM 1. Under the condition that $m$ is even, for every eventually positive solution $\left\{x_{n}\right\}$ of $(1)$, there is some integer $j \in\{1,2, \ldots, m / 2\}$ such that $\left\{x_{n}\right\}$ belongs to one of the classes $E_{j}(\infty, *), E_{j}(\infty, 0)$, or $E_{j}(*, 0)$. Under the condition that $m$ is odd, for every eventually positive solution $\left\{x_{n}\right\}$ of $(1)$, either there is $j \in\{1,2, \ldots,(m-1) / 2\}$ such that it belongs to one of the classes $O_{j}(\infty, *), O_{j}(\infty, 0), O_{j}(*, 0)$, or else it converges.

ProOf. Assume that $\left\{x_{n}\right\}$ is an eventually positive solution of (1). Then the associated sequence $\left\{z_{n}\right\}$ of $\left\{x_{n}\right\}$ is eventually positive, furthermore, in view of $(1),\left\{\Delta^{m} z_{n}\right\}$ is eventually negative.

Suppose $m$ is even. Then by Lemma 3, there exist integers $n_{1}$ and $N=2 j-1$, where $j \in\{1, \ldots, m / 2\}$, such that

$$
\Delta^{k} z_{n}>0, \quad n=n_{1}, n_{1}+1, \ldots ; k=0,1, \ldots, N-1,
$$

and

$$
(-1)^{k+1} \Delta^{k} z_{n}>0, \quad n=n_{1}, n_{1}+1, \ldots ; k=N, N+1, \ldots, m-1 .
$$

In particular, $\Delta^{2 j-2} z_{n}>0, \Delta^{2 j-1} z_{n}>0$ and $\Delta^{2 j} z_{n}<0$ for $n \geq n_{1}$. Thus

$$
0 \leq \lambda_{2 j-1} \equiv \lim _{n \rightarrow \infty} \Delta^{2 j-1} z_{n}<\infty \quad \text { and } \quad 0<\lambda_{2 j-2} \equiv \lim _{n \rightarrow \infty} \Delta^{2 j-2} z_{n} \leq \infty .
$$

If $\lambda_{2 j-1}>0$, then by Stolz's theorem, we have

$$
\lim _{n \rightarrow \infty} \frac{z_{n}}{n^{2 j-1}}=\lim _{n \rightarrow \infty} \frac{\Delta z_{n}}{(2 j-1) n^{2 j-2}}=\cdots=\lim _{n \rightarrow \infty} \frac{\Delta^{2 j-1} z_{n}}{(2 j-1) !}=\frac{\lambda_{2 j-1}}{(2 j-1) !}<\infty .
$$

Since $0 \leq x_{n} / n^{2 j-1} \leq z_{n} / n^{2 j-1}$, we see that $x_{n} / n^{2 j-1}$ is bounded for $n \geq n_{1}$. In view of Lemma 1, we have

$$
\lim _{n \rightarrow \infty} \frac{x_{n}}{n^{2 j-1}}=\frac{\lambda_{2 j-1}}{(2 j-1) !(1+p)} \neq 0
$$


It follows that

$$
\lim _{n \rightarrow \infty} \frac{x_{n}}{n^{2 j-2}}=\infty
$$

and hence $\left\{x_{n}\right\} \in E_{j}(\infty, *)$.

If $\lambda_{2 j-1}=0$ and $\lambda_{2 j-2}=\infty$, then by Stolz's theorem, it is easy to see that

$$
\lim _{n \rightarrow \infty} \frac{z_{n}}{n^{2 j-1}}=0 \quad \text { and } \quad \lim _{n \rightarrow \infty} \frac{z_{n}}{n^{2 j-2}}=\infty
$$

In view of Lemma 1, we see further that

$$
\lim _{n \rightarrow \infty} \frac{x_{n}}{n^{2 j-1}}=0 \quad \text { and } \quad \limsup _{n \rightarrow \infty} \frac{x_{n}}{n^{2 j-2}}=\infty .
$$

Hence $\left\{x_{n}\right\}$ belongs to $E_{j}(\infty, 0)$.

If $\lambda_{2 j-1}=0$ and $0<\lambda_{2 j-2}<\infty$, then by Stolz's theorem, we have

$$
\lim _{n \rightarrow \infty} \frac{z_{n}}{n^{2 j-2}}=\frac{\lambda_{2 j-2}}{(2 j-1) !} \neq 0 .
$$

In view of Lemma 1 , we see that

$$
\lim _{n \rightarrow \infty} \frac{x_{n}}{n^{2 j-2}}=\frac{\lambda_{2 j-2}}{(2 j-1) !(1+p)} \neq 0 .
$$

It follows that $\lim _{n \rightarrow \infty} x_{n} / n^{2 j-1}=0$. Hence $\left\{x_{n}\right\}$ belongs to $E_{j}(*, 0)$.

When $m$ is odd, in view of Lemma 3 , there is an even integer $t \in\{0,1, \ldots, m-1\}$ such that for each $s \in\{0,1, \ldots, t\}, \Delta^{s} x_{n}>0$ for all large $n$, and for each $s \in\{t+1$, $\ldots, m-1\},(-1)^{s-t} \Delta^{s} x_{n}>0$ for all large $k$. In case $t \in\{1,2, \ldots, m-1\}$, the proof is similar to that given above. In case $t=0$, then $x_{n}>0, \Delta x_{n}<0$ and $\Delta^{2} x_{n}>0$ for all large $n$. It follows that $\left\{x_{n}\right\}$ converges to some nonnegative constant. The proof is complete.

\section{Existence criteria when $m$ is even}

Eventually positive (and by analogy eventually negative) solutions of (1) have been classified according to Theorem 1 . We remark, however, that there is an uncertainty involved, namely, the integer $j$ which is needed in the definitions of $E_{j}$ and $O_{j}$. We now justify our classification schemes by finding existence criteria for solutions in $E_{j}$ and $O_{j}$. 
THEOREM 2. Suppose that $m$ is even. Suppose further that $f$ is superlinear or sublinear. If equation (1) has an eventually positive solution in $E_{j}(\infty, *)$ for some $j \in\{1,2, \ldots, m / 2\}$, then there is a constant $C>0$ such that

$$
\sum_{n=0}^{\infty} n^{m-2 j} f\left(n, C(n-\delta)^{2 j-1}\right)<\infty .
$$

The converse is also true.

PROOF. Let $\left\{x_{n}\right\}$ be an eventually positive solution in $E_{j}(\infty, *)$ so that $\lim _{n \rightarrow \infty} x_{n} /$ $n^{2 j-2}=\infty$ and $\lim _{n \rightarrow \infty} x_{n} / n^{2 j-1}=a>0$. Then its associated sequence $\left\{z_{n}\right\}$ is eventually positive. Since $\left\{\Delta^{m} z_{n}\right\}$ is eventually negative in view of (1), we may apply Lemma 3 and conclude that $\left\{\Delta^{i} z_{n}\right\}$ is eventually monotonic for each $i \in$ $\{1,2, \ldots, m-1\}$. Let $n_{1}$ be an integer such that

$$
\frac{a n^{2 j-1}}{2} \leq x_{n} \leq \frac{3 a n^{2 j-1}}{2}
$$

for $n \geq n_{1}$. Note first that

$$
\lim _{n \rightarrow \infty} \Delta^{2 j-1} z_{n}=(2 j-1) !(1+p) a,
$$

which holds since by the theorem of Stolz

$$
\lim _{n \rightarrow \infty} \frac{z_{n}}{n^{2 j-1}}=\cdots=\lim _{n \rightarrow \infty} \frac{\Delta^{2 j-1} z_{n}}{(2 j-1) !}=(1+p) a .
$$

Next, since $\left\{\Delta^{i} z_{n}\right\}$ is eventually monotonic for $i \in\{2 j, 2 j+1, \ldots, m-1\}$, we see further that

$$
\lim _{n \rightarrow \infty} \Delta^{2 j} z_{n}=\lim _{n \rightarrow \infty} \Delta^{2 j+1} z_{n}=\cdots=\lim _{n \rightarrow \infty} \Delta^{m-1} z_{n}=0 .
$$

Thus by summing (1) successively, we obtain

$$
\Delta^{2 j} z_{n}=(-1)^{m-2 j+1} \sum_{i=n}^{\infty} \frac{(i-n+1) \cdots(i-n+m-2 j-1)}{(m-2 j-1) !} f\left(i, x_{i-\delta}\right)
$$

for $n \geq n_{1}$. Summing again, we obtain

$$
\begin{aligned}
& \Delta^{2 j-1} z_{n_{1}}-(2 j-1) !(1+p) a \\
& \quad=(-1)^{m-2 j+1} \sum_{i=n_{1}}^{\infty} \frac{\left(i-n_{1}+1\right) \cdots\left(i-n_{1}+m-2 j\right)}{(m-2 j) !} f\left(i, x_{i-\delta}\right),
\end{aligned}
$$


which implies

$$
\sum_{i=0}^{\infty} i^{m-2 j} f\left(i, x_{i-\delta}\right)<\infty .
$$

If $f$ is superlinear, then $f\left(n, a n^{2 j-1} / 2\right) \leq f\left(n, x_{n}\right)$ for $n \geq n_{1}$. Hence

$$
\sum_{i=0}^{\infty} i^{m-2 j} f\left(i, \frac{a}{2}(i-\delta)^{2 j-1}\right)<\infty .
$$

Similarly, if $f$ is sublinear, then

$$
\sum_{i=0}^{\infty} i^{m-2 j} f\left(i, \frac{3 a}{2}(i-\delta)^{2 j-1}\right)<\infty .
$$

Next, we assume that (2) holds for some $j \in\{1,2, \ldots, m / 2\}$ and $C>0$. Let $D=C / 2$ if $f$ is superlinear and let $D=C$ if $f$ is sublinear. Let

$$
\Gamma(n)=n^{2 j-1}, \quad n=0,1, \ldots
$$

We need to consider the cases $p=0,0<p<1$ and $p>1$. If $p \in(0,1)$, we have

$$
\lim _{n \rightarrow \infty} p \frac{\Gamma(n)}{\Gamma(n-\tau-\delta)}=p
$$

and

$$
\lim _{n \rightarrow \infty} \frac{\Gamma(n-\tau)}{\Gamma(n)}=1>1-\frac{1-p}{4 p} .
$$

Choose $p_{1} \in(p, 1)$ and $M \geq \tau+\delta$ such that

$$
\frac{\Gamma(M)}{\Gamma(M-\tau-\delta)}<2,
$$

for $n \geq M$, and

$$
\sum_{i=M}^{\infty} \frac{(i-M+1) \cdots(i-M+m-2 j)}{(m-2 j) !} f\left(i, C(i-\delta)^{2 j-1}\right)<\frac{(1-p) D}{8}
$$

Let $N=M-\tau-\delta$. Then $p \Gamma(M) / \Gamma(N)<p_{1}$ by (3). We introduce the Banach space $B$ of all real sequences $x=\left\{x_{n}\right\}_{n=N}^{\infty}$ endowed with the usual operations and the norm

$$
\|x\|=\sup _{n \geq N} \frac{\left|x_{n}\right|}{\Gamma^{2}(n)}
$$


and let $\Omega$ be the subset of $B$ defined by

$$
\Omega=\left\{\left\{x_{n}\right\} \in B \mid D \Gamma(n) \leq x_{n} \leq 2 D \Gamma(n), n \geq N\right\} .
$$

Let $T: \Omega \rightarrow \Omega$ be defined as follows: for $x=\left\{x_{n}\right\} \in \Omega$,

$$
(T x)_{n}=D \Gamma(M), \quad N \leq n<M,
$$

and

$$
\begin{aligned}
(T x)_{n}= & \frac{3 D(1+p)}{2} \Gamma(n)-p x_{n-\mathfrak{r}} \\
& +\sum_{i_{m-1}=M}^{n-1} \sum_{i_{m-2}=M}^{i_{m-1}-1} \ldots \sum_{i_{m-2 j+1}=M}^{i_{m-2 j+2}-1} H_{i_{m-2 j+2}}^{m-2 j}(i) f\left(i, x_{i-\delta}\right), \quad n \geq M,
\end{aligned}
$$

where we have employed the convenient notation

$$
H_{t}^{s}(i) u_{i} \equiv \sum_{i=t}^{\infty} \frac{(i-t+1) \cdots(i-t+s)}{s !} u_{i} .
$$

The subset $\Omega$ is bounded, convex and closed. We assert further that $T$ maps $\Omega$ into $\Omega, T$ is continuous, and that $T \Omega$ is uniformly Cauchy. Indeed, if $x=\left\{x_{n}\right\} \in \Omega$, then from the definitions of $M, N$ and from (3) as well as (4), we see that

$$
\begin{aligned}
D \Gamma(n) & <D \Gamma(M)=(T x)_{n}<2 D \Gamma(n), \quad N \leq n<M, \\
(T x)_{n} & \geq \frac{3 D(1+p)}{2} \Gamma(n)-p x_{n-\tau} \geq \frac{3 D(1+p)}{2} \Gamma(n)-2 p D \Gamma(n-\tau) \\
& \geq \frac{3 D(1+p)}{2} \Gamma(n)-2 p D \Gamma(n) \geq D \Gamma(n), \quad n \geq M,
\end{aligned}
$$

and

$$
\begin{aligned}
(T x)_{n} & \leq \frac{3 D(1+p)}{2} \Gamma(n)-p x_{n-\tau}+\frac{(1-p) D}{8} \Gamma(n) \\
& \leq \frac{3 D(1+p)}{2} \Gamma(n)-D p\left(1-\frac{1-p}{4 p}\right) \Gamma(n)+\frac{(1-p) D}{8} \Gamma(n) \\
& \leq \frac{15 D+D p}{8} \Gamma(n) \leq 2 D \Gamma(n), \quad n \geq M .
\end{aligned}
$$

This shows that $T x \in \Omega$. Next, let $\left\{x^{(k)}\right\}_{k=1}^{\infty}$ be a sequence in $\Omega$ such that $\lim _{k \rightarrow \infty} x^{(k)}=$ $x$. Since $\Omega$ is closed, $x \in \Omega$. Furthermore, for $n \geq M$, we have

$$
\frac{\left|\left(T x^{(k)}\right)_{n}-(T x)_{n}\right|}{\Gamma^{2}(n)} \leq p \frac{\left|x_{n-\tau}^{(k)}-x_{n-\tau}\right|}{\Gamma^{2}(n)}
$$




$$
\begin{aligned}
& +\frac{1}{\Gamma^{2}(n)} \sum_{i_{m-1}=M}^{n-1} \sum_{i_{m-2}=M}^{i_{m-1}-1} \ldots \sum_{i_{m-2 j+1}=M}^{i_{m-2 j+2}-1} H_{i_{m-2 j+1}}^{m-2 j}(i)\left|f\left(i, x_{i-\delta}^{(k)}\right)-f\left(i, x_{i-\delta}\right)\right| \\
\leq & p\left\|x^{(k)}-x\right\|+\frac{1}{\Gamma^{2}(n)} \frac{(n-M)^{2 j-1}}{(2 j-1) !} H_{M}^{m-2 j}(i)\left|f\left(i, x_{i-\delta}^{(k)}\right)-f\left(i, x_{i-\delta}\right)\right| \\
\leq & p\left\|x^{(k)}-x\right\|+\frac{1}{\Gamma(n)} \frac{1}{(2 j-1) !} H_{M}^{m-2 j}(i)\left|f\left(i, x_{i-\delta}^{(k)}\right)-f\left(i, x_{i-\delta}\right)\right| .
\end{aligned}
$$

Since

$$
\left|f\left(i, x_{i-\delta}^{(k)}\right)-f\left(i, x_{i-\delta}\right)\right| \leq f\left(i, x_{i-\delta}^{(k)}\right)+f\left(i, x_{i-\delta}\right) \leq 4 f\left(i, C(i-\delta)^{2 j-1}\right)
$$

and since $f$ is continuous in the second variable, by the Lebesgue's dominated convergence theorem, we see that

$$
\lim _{k \rightarrow \infty}\left\|T x^{(k)}-T x\right\|=\lim _{k \rightarrow \infty} \sup _{n \geq N} \frac{\left|\left(T x^{(k)}\right)_{n}-(T x)_{n}\right|}{\Gamma^{2}(n)}=0 .
$$

This shows that $T$ is continuous.

Finally, we assert that $T \Omega$ is uniformly Cauchy. To see this, we have to show that given any $\varepsilon>0$ there exists an integer $P$ such that for $t, n \geq P$

$$
\left|\frac{(T x)_{t}}{\Gamma^{2}(t)}-\frac{(T x)_{n}}{\Gamma^{2}(n)}\right|<\varepsilon
$$

for any $\left\{x_{n}\right\} \in \Omega$. Indeed, assuming without loss of generality that $t>n$, we have

$$
\begin{aligned}
\mid \frac{(T x)_{t}}{\Gamma^{2}(t)} & -\frac{(T x)_{n}}{\Gamma^{2}(n)} \mid \leq \frac{3 D(1+p)}{2 \Gamma(t)}+\frac{3 D(1+p)}{2 \Gamma(n)}+p\left(\frac{x_{t-\tau}}{\Gamma^{2}(t)}+\frac{x_{n-t}}{\Gamma^{2}(n)}\right) \\
& +\frac{1}{\Gamma^{2}(n)} \sum_{i_{m-1}=M}^{t-1} \sum_{i_{m-2}=M}^{i_{m-1}-1} \cdots \sum_{i_{m-2 j+1}=M}^{i_{m-2 j+2}-1} H_{i_{m-2 j+2}}^{m-2 j}(i)\left(f\left(i, x_{i-\delta}^{(k)}\right)+f\left(i, x_{i-\delta}\right)\right) \\
& \leq \frac{3 D(1+p)}{\Gamma(n)}+\frac{4 D p}{\Gamma(n)}+\frac{(1-p) D}{8}\left(\frac{1}{\Gamma(t)}+\frac{1}{\Gamma(n)}\right) \\
& \leq \frac{3 D(1+p)}{\Gamma(n)}+\frac{4 D p}{\Gamma(n)}+\frac{(1-p) D}{4 \Gamma(n)} \\
& \leq \frac{6 D(1+p)+8 D p+(1-p) D}{4 \Gamma(n)} \leq \frac{5 D}{\Gamma(n)}
\end{aligned}
$$

Since $\Gamma(n)$ tends to infinity as $n$ tends to infinity, we see that our assertion holds.

As a consequence, we may now apply Lemma 5 to conclude that $T$ has a fixed point $x^{*}=\left\{x_{n}^{*}\right\}$ in $\Omega$. In view of (5), we now see that

$$
x_{n}^{*}+p x_{n-\tau}^{*}=\frac{3 D(1+p)}{2} \Gamma(n)+\sum_{i_{m-1}=M}^{n-1} \sum_{i_{m-2}=M}^{i_{m-1}-1} \ldots \sum_{i_{m-2 j+1}=M}^{i_{m-2 j+2-1}} H_{i_{m-2 j+2}}^{m-2 j}(i) f\left(i, x_{i-\delta}^{*}\right) \text {. }
$$


By taking differences on both sides of the above equality, we see that $x^{*}$ is a solution of (1). Furthermore, by applying the theorem of Stolz, we also see that

$$
\begin{aligned}
\lim _{n \rightarrow \infty} & \frac{x_{n}^{*}+p x_{n-\tau}^{*}}{n^{2 j-1}} \\
= & \frac{3(1+p) D}{2}+\lim _{n \rightarrow \infty} \frac{1}{n^{2 j-1}} \sum_{i_{m-1}=M}^{n-1} \sum_{i_{m-2}=M}^{i_{m-1}-1} \cdots \sum_{i_{m-2 j+1}=M}^{i_{m-2 j+2}-1} H_{i_{m-2 j+2}^{m-2 j}}^{m}(i) f\left(i, x_{i-\delta}^{*}\right) \\
& =\frac{3(1+p) D}{2}+\lim _{n \rightarrow \infty} \frac{1}{(2 j-1) !} H_{n}^{m-2 j}(i) f\left(i, x_{i-\delta}^{*}\right)=\frac{3(1+p) D}{2} .
\end{aligned}
$$

Finally, by Lemma 1, we have

$$
\lim _{n \rightarrow \infty} \frac{x_{n}^{*}}{n^{2 j-1}}=\frac{3 D}{2} \neq 0
$$

which also implies that

$$
\lim _{n \rightarrow \infty} \frac{x_{n}^{*}}{n^{2 j-2}}=\infty
$$

In other words, we have found a solution in $E_{j}(\infty, *)$ for the case $p \in(0,1)$.

For the case $p=0$, we modify the definition of $M$ in the above arguments so that

$$
H_{M}^{m-2 j}(i) f\left(i, C(i-\delta)^{2 j-1}\right)<\frac{D}{8},
$$

and modify the definition of $T$ so that

$$
(T x)_{n}=\frac{3 D}{2} \Gamma(n)+\sum_{i_{m-1}=M}^{n-1} \sum_{i_{m-2}=M}^{i_{m-1}-1} \cdots \sum_{i_{m-2 j+1}=M}^{i_{m-2 j+2}-1} H_{i_{m-2 j+2}}^{m-2 j}(i) f\left(i, x_{i-\delta}\right)
$$

for $n \geq M$. Then under the same $N, B$, and $\Omega$, we may show that (1) has a solution in $E_{j}(\infty, *)$.

For the case $p>1$, we first choose $p_{1}$ such that $0<1 / p<p_{1}<1$. We also change the definition of $M$ so that $M \geq \tau+\delta$ and

$$
\begin{aligned}
& \frac{1}{p} \frac{\Gamma^{2}(n+\tau)}{\Gamma^{2}(n-\tau-\delta)}<p_{1}<1, \quad n \geq M, \\
& \frac{\Gamma(n+\tau)}{\Gamma(n)}<1+\frac{p-1}{4}, \quad n \geq M,
\end{aligned}
$$

and

$$
H_{M}^{m-2 j}(i) f\left(i, C(i-\delta)^{2 j-1}\right)<\frac{(p-1) D}{8}
$$


Finally, we modify the definition of $T$ so that

$$
(T x)_{n}=\frac{3 D(1+p)}{2 p} \Gamma(n)-\frac{1}{p} \frac{\Gamma(n)}{\Gamma(M)} x_{M+\tau}, \quad N \leq n<M,
$$

and

$$
\begin{aligned}
(T x)_{n}= & \frac{3 D(1+p)}{2 p} \Gamma(n)-\frac{1}{p} x_{n+\tau} \\
& +\frac{1}{p} \sum_{i_{m-1}=M+\tau}^{n+\tau-1} \sum_{i_{m-2}=M}^{i_{m-1}-1} \ldots \sum_{i_{m-2 j+1}=M}^{i_{m-2 j+2}-1} H_{i_{m-2 j+2}^{m-2 j}}^{m}(i) f\left(i, x_{i-\delta}\right), \quad n \geq M .
\end{aligned}
$$

The same reasoning described above will lead to the conclusion that (1) has a solution in $E_{j}(\infty, *)$. The proof is complete.

Next we turn our attention to solutions in $E_{j}(*, 0)$.

THEOREM 3. Suppose that $m$ is even. Suppose further that $f$ is superlinear or sublinear. If equation (1) has an eventually positive solution in $E_{j}(*, 0)$ for some $j \in\{1,2, \ldots, m / 2\}$, then there is a constant $C>0$ such that

$$
\sum_{n=0}^{\infty} n^{m-2 j+1} f\left(n, C(n-\delta)^{2 j-2}\right)<\infty
$$

The converse is also true.

Proof. If equation (1) has an eventually positive solution in $E_{j}(*, 0)$, then by modifying the arguments in the proof of Theorem 2 , it is not difficult to see that (6) holds for some positive constant $C$. The proof of the converse is also similar to that of Theorem 2. We first retain the definition of the Banach space $B$ and its subspace $\Omega$ in the proof of Theorem 2. We then change the definition of $\Gamma(n)$ to $\Gamma(n)=n^{2 j-2}$ for $n \geq 0$. Furthermore, for the case $p \in(0,1)$, we choose $p_{1} \in(p, 1)$ and $M \geq \tau+\delta$ such that

$$
p \frac{\Gamma(n)}{\Gamma(n-\tau-\delta)}<p_{1}, \quad \frac{\Gamma(n-\tau)}{\Gamma(n)}>1-\frac{1-p}{4 p}
$$

for $n \geq M$, and

$$
\sum_{i=M}^{\infty} \frac{(i-M+1) \cdots(i-M+m-2 j+1)}{(m-2 j+1) !} f\left(i, C(i-\delta)^{2 j-2}\right)<\frac{(1-p) D}{8}
$$

We modify the definition of the operator $T$ by

$$
(T x)_{n}=D \Gamma(M), \quad N \leq n<M,
$$


and

$$
\begin{aligned}
(T x)_{n}= & \frac{3 D(1+p)}{2} \Gamma(n)-p x_{n-\tau} \\
& +\sum_{i_{m-1}=M}^{n-1} \sum_{i_{m-2}=M}^{i_{m-1}-1} \cdots \sum_{i_{m-2 j+2}=M}^{i_{m-2 j+3}-1} H_{i_{m-2 j+3}^{m-2 j+1}}^{m}(i) f\left(i, x_{i-\delta}\right), \quad n \geq M .
\end{aligned}
$$

For the case where $p=0$, we modify the definition of $M$ in Theorem 2 so that

$$
H_{M}^{m-2 j-1}(i) f\left(i, C(i-\delta)^{2 j-2}\right)<\frac{D}{8} .
$$

Let $N=M-\tau-\delta$. We modify the definition of $T$ by

$$
(T x)_{n}=D \Gamma(M), \quad N \leq n<M,
$$

and

$$
(T x)_{n}=\frac{3 D}{2} \Gamma(n)+\sum_{i_{m-1}=M}^{n-1} \sum_{i_{m-2}=M}^{i_{m-1}-1} \cdots \sum_{i_{m-2 j+2}=M}^{i_{m-2 j+3}-1} H_{i_{m-2 j+3}^{m-1}}^{m-2 j+1}(i) f\left(i, x_{i-\delta}\right)
$$

for $n \geq M$.

Finally for the case $p>1$, we choose $p_{1} \in(1 / p, 1)$ and $M \geq \tau+\delta$ such that

$$
\begin{aligned}
& \frac{1}{p} \frac{\Gamma^{2}(n+\tau)}{\Gamma^{2}(n-\tau-\delta)}<p_{1}<1, \quad n \geq M, \\
& \frac{\Gamma(n+\tau)}{\Gamma(n)}<1+\frac{p-1}{4}, \quad n \geq M,
\end{aligned}
$$

and

$$
H_{M}^{m-2 j-1}(i) f\left(i, C(i-\delta)^{2 j-2}\right)<\frac{(p-1) D}{8} .
$$

We also modify the definition of $T$ so that

$$
(T x)_{n}=\frac{3 D(1+p)}{2 p} \Gamma(n)-\frac{1}{p} \frac{\Gamma(n)}{\Gamma(M)} x_{M+\tau}, \quad N \leq n<M,
$$

and

$$
\begin{aligned}
(T x)_{n}= & \frac{3 D(1+p)}{2 p} \Gamma(n)-\frac{1}{p} x_{n+\tau} \\
& +\frac{1}{p} \sum_{i_{m-1}=M+\tau}^{n+\tau-1} \sum_{i_{m-2}=M}^{i_{m-1}-1} \cdots \sum_{i_{m-2 j+2}=M}^{i_{m-2 j+3}-1} H_{i_{m-2 j+3}^{m-2 j+1}}(i) f\left(i, x_{i-\delta}\right), \quad n \geq M .
\end{aligned}
$$

Then the same reasoning described in the proof of Theorem 2 will lead to the conclusion that $(1)$ has a solution in $E_{j}(*, 0)$. 
Next we turn our attention to solutions in $E_{j}(\infty, 0)$.

THEOREM 4. Suppose that $m$ is even. Suppose further that for each fixed $n \geq 0$, $f(n, x)$ is nonincreasing in $x$ over the interval $(0, \infty)$. If equation (1) has an eventually positive solution in $E_{j}(\infty, 0)$ for some $j \in\{1,2, \ldots, m / 2\}$, then

$$
\sum_{n=0}^{\infty} n^{m-2 j} f\left(n, a(n-\delta)^{2 j-1}\right)<\infty
$$

for every $a>0$, and

$$
\sum_{n=0}^{\infty} n^{m-2 j+1} f\left(n, b(n-\delta)^{2 j-2}\right)=\infty
$$

for every $b>0$. Conversely, if (7) holds for every $a>0$ and

$$
\sum_{n=0}^{\infty} n^{m-2 j} f\left(n, C(n-\delta)^{2 j-2}\right)<\infty
$$

for some $C>0$, then equation (1) has a positive solution in $E_{j}(\infty, 0)$.

PROOF. Let $\left\{x_{n}\right\}$ be an eventually positive solution in $E_{j}(\infty, 0)$ so that

$$
\limsup _{n \rightarrow \infty} \frac{x_{n}}{n^{2 j-2}}=\infty \text {, }
$$

and

$$
\lim _{n \rightarrow \infty} \frac{x_{n}}{n^{2 j-1}}=0
$$

For any $a>0$ and $b>0$, there exists $n_{1} \geq 0$ such that

$$
b n^{2 j-2} \leq x_{n} \leq a n^{2 j-1}, \quad n \geq n_{1},
$$

which, in view of the decreasing property of $f$, implies that

$$
f\left(n, a n^{2 j-1}\right) \leq f\left(n, x_{n}\right) \leq f\left(n, b n^{2 j-2}\right), \quad n \geq n_{1} .
$$

Now we may follow the arguments in the proof of Theorem 2 and conclude that (7) holds for any $a>0$. Next, note that the associated sequence $\left\{z_{n}\right\}$ of $\left\{x_{n}\right\}$ satisfies

$$
\lim _{n \rightarrow \infty} \Delta^{2 j-2} z_{n}=\infty
$$


which holds since, by the theorem of Stolz,

$$
\limsup _{n \rightarrow \infty} \frac{x_{n}}{n^{2 j-2}} \leq \limsup _{n \rightarrow \infty} \frac{z_{n}}{n^{2 j-2}}=\lim _{n \rightarrow \infty} \frac{z_{n}}{n^{2 j-2}}=\cdots=\lim _{n \rightarrow \infty} \frac{\Delta^{2 j-2} z_{n}}{(2 j-2) !}=\infty .
$$

Since $\left\{\Delta^{i} z_{n}\right\}$ is eventually monotonic for $i \in\{2 j-1,2 j, \ldots, m-1\}$, we see further that

$$
\lim _{n \rightarrow \infty} \Delta^{2 j-1} z_{n}=\cdots=\lim _{n \rightarrow \infty} \Delta^{n-1} z_{n}=0
$$

Thus by summing (1) successively, we obtain

$$
\Delta^{2 j-1} z_{n}=(-1)^{m-2 j} \sum_{i=n}^{\infty} \frac{(i-n+1) \cdots(i-n+m-2 j)}{(m-2 j) !} f\left(i, x_{i-\delta}\right)
$$

for $n \geq n_{1}$. Summing again, we obtain

$$
\begin{aligned}
\Delta^{2 j-2} z_{n}-\Delta^{2 j-2} z_{n_{1}} & \\
& =(-1)^{m-2 j} \sum_{i=n_{1}}^{n-1} \sum_{k=i}^{\infty} \frac{(k-i+1) \cdots(k-i+m-2 j)}{(m-2 j) !} f\left(k, x_{k-\delta}\right) \\
& \leq \sum_{n=n_{1}}^{\infty} \frac{\left(n-n_{1}+1\right) \cdots\left(n-n_{1}+m-2 j+1\right)}{(m-2 j+1) !} f\left(n, x_{n-\delta}\right),
\end{aligned}
$$

which, since $\lim _{n \rightarrow \infty} \Delta^{2 j-2} z_{n}=\infty$ and $f\left(n, x_{n}\right) \leq f\left(n, b n^{2 j-2}\right)$, implies

$$
\sum_{n=n_{1}+\delta}^{\infty} n^{m-2 j+1} f\left(n, b(n-\delta)^{2 j-2}\right)=\infty
$$

for every $b>0$. This shows that

$$
\sum_{n=0}^{\infty} n^{m-2 j+1} f\left(n, b(n-\delta)^{2 j-2}\right)=\infty
$$

for every $b>0$.

Conversely, assuming that (8) holds for every $b>0$ and (9) holds for some $C>0$, we may proceed as in the proof of Theorem 2. We first retain the definitions of the Banach space $B$ and its subspace $\Omega$. Then we change the definition of $\Gamma(n)$ to $\Gamma(n)=n^{2 j-2}$ for $n \geq 0$. We have three cases to consider: $p=0,0<p<1$ and $p>1$. We will only consider the case $p \in(0,1)$, for the other two cases can be proved in similar manners. Choose $p_{1} \in(p, 1)$ and $M \geq \tau+\delta$ such that

$$
\frac{\Gamma(n)}{\Gamma(n-\tau-\delta)}<p_{1}, \quad \frac{\Gamma(n-\tau)}{\Gamma(n)}>1-\frac{1-p}{4 p}
$$


for $n \geq M$, and

$$
\sum_{i=M}^{\infty} \frac{(i-M+1) \cdots(i-M+m-2 j)}{(m-2 j) !} f\left(i, C(i-\delta)^{2 j-2}\right)<\frac{(1-p) D}{8} .
$$

We modify the definition of the operator $T$ by

$$
(T x)_{n}=D \Gamma(M), \quad N \leq n<M,
$$

and

$$
\begin{aligned}
(T x)_{n}= & \frac{3 D(1+p)}{2} \Gamma(n)-p x_{n-\tau} \\
& +\sum_{i_{m-1}=M}^{n-1} \sum_{i_{m-2}=M}^{i_{m}-1} \ldots \sum_{i_{m-2 j+3}=M}^{i_{m-2 j+4}-1} H_{i_{m-2 j+4}}^{m-2 j}(i) f\left(i, x_{i-\delta}\right), \quad n \geq M
\end{aligned}
$$

Using similar argument as in the proof of Theorem 2 we conclude that (1) has a solution $x^{*}$ in $\Omega$. Furthermore, applying the theorem of Stolz, we see that

$$
\lim _{n \rightarrow \infty} \frac{x^{*}+p x_{n-\tau}^{*}}{n^{2 j-2}}=\frac{3(1+p) D}{2}+\lim _{n \rightarrow \infty} \frac{1}{(2 j-2) !} \sum_{k=M}^{n-1} H_{k}^{m-2 j}(i) f\left(i, x_{i-\delta}^{*}\right)
$$

and

$$
\lim _{n \rightarrow \infty} \frac{x^{*}+p x_{n-\tau}^{*}}{n^{2 j-1}}=\lim _{n \rightarrow \infty} \frac{1}{(2 j-1) !} H_{n}^{m-2 j}(i) f\left(i, x_{i-\delta}^{*}\right)=0 .
$$

Hence, by Lemma 1, we have

$$
\lim _{n \rightarrow \infty} \frac{x_{n}^{*}}{n^{2 j-1}}=0
$$

Since the associated sequence $\left\{z_{n}^{*}\right\}$ of $\left\{x_{n}^{*}\right\}$ satisfies $\Delta^{2 j-2} z^{*}>0$ and $\Delta^{2 j-1} z^{*}<0$ for all large $n$, the sequence $\left\{\Delta^{2 j-2} z_{n}^{*}\right\}$ is eventually positive and increasing. Thus $\left\{\Delta^{2 j-2} z_{n}^{*}\right\}$ either converges to some positive limit or diverges to $\infty$. If the first case holds, then

$$
\lim _{n \rightarrow \infty} \frac{z_{n}^{*}}{n^{2 j-2}}=\theta
$$

for some constant $\theta$, and hence by Lemma 1 ,

$$
\lim _{n \rightarrow \infty} \frac{x_{n}^{*}}{n^{2 j-2}}=\frac{\theta}{1+p} .
$$

But then $x^{*}$ will belong to $E_{j}(*, 0)$ so that $(6)$ holds for some constant $C>0$. This is contrary to the assumption that (8) holds for every $b>0$. In other words, $x^{*}$ either belongs to $E_{j}(*, 0)$ or $E_{j}(\infty, 0)$. The latter case is, however, excluded by $(10)$. The proof is complete. 


\section{Existence criteria when $m$ is odd}

For the case where $m$ is odd, we have four results. The first three correspond to Theorem 2, Theorem 3 and Theorem 4 in the last section. The proofs of these results are similar in nature to those above and hence are sketched or omitted.

THEOREM 5. Suppose that $m$ is odd. Suppose further that $f$ is superlinear or sublinear. If equation (1) has an eventually positive solution in $O_{j}(\infty, *)$ for some $j \in\{1,2, \ldots,(m-1) / 2\}$, then there is a constant $C>0$ such that

$$
\sum_{n=0}^{\infty} n^{m-2 j-1} f\left(n, C(n-\delta)^{2 j}\right)<\infty .
$$

The converse is also true.

The proof is again similar to that of Theorem 2. In particular, to show the converse, we first retain the definitions of the Banach space $B$ and its subspace $\Omega$ in the proof of Theorem 2. We then change the definition of $\Gamma(n)$ to $\Gamma(n)=n^{2 j}$ for $n \geq 0$. Furthermore, for the case $p \in[0,1)$, we modify the definition of $T$ so that

$$
\begin{aligned}
(T x)_{n}= & D \Gamma(M), \quad N \leq n<M, \\
(T x)_{n}= & \frac{3 D(1+p)}{2} T(n)-p x_{n-\tau} \\
& +\sum_{i_{m-1}=M}^{n-1} \sum_{i_{m-2}=M}^{i_{m-1}-1} \cdots \sum_{i_{m-2 j}=M}^{i_{m-2 j+1}-1} H_{i_{m-2 j+1}}^{m-2 j-1}(i) f\left(i, x_{i-\delta}\right), \quad n \geq M ;
\end{aligned}
$$

and for the case $p>1$, we modify the definition of $T$ so that

$$
\begin{aligned}
(T x)_{n}= & \frac{3 D(1+p)}{2 p} \Gamma(n)-\frac{1}{p} \frac{\Gamma(n)}{\Gamma(M)} x_{M+\tau}, \quad N \leq n<M, \\
(T x)_{n}= & \frac{3 D(1+p)}{2 p} \Gamma(n)-\frac{1}{p} x_{n+\tau} \\
& +\frac{1}{p} \sum_{i_{m-1}=M+\tau}^{n+\tau-1} \sum_{i_{m-2}=M}^{i_{m-1}-1} \cdots \sum_{i_{m-2 j}=M}^{i_{m-2 j+1}-1} H_{i_{m-2 j+1}}^{m-2 j-1}(i) f\left(i, x_{i-\delta}\right), \quad n \geq M .
\end{aligned}
$$

Then the same reasoning described in the proof of Theorem 2 will lead to the conclusion that (1) has a solution in $O_{j}(\infty, *)$.

THEOREM 6. Suppose that $m$ is odd. Suppose further that $f$ is superlinear or sublinear. If equation (1) has an eventually positive solution in $O_{j}(*, 0)$ for some 
$j \in\{1,2, \ldots,(m-1) / 2\}$, then there is a constant $C>0$ such that

$$
\sum_{n=0}^{\infty} n^{m-2 j} f\left(n, C(n-\delta)^{2 j-1}\right)<\infty
$$

The converse is also true.

The proof of this result is similar to those of Theorem 2, Theorem 3 and Theorem 4 , and is thus omitted.

THEOREM 7. Suppose that $m$ is odd. Suppose further that for each fuxed $n \geq 0$, $f(n, x)$ is nonincreasing in $x$ over the interval $(0, \infty)$. If equation (1) has an eventually positive solution in $O_{j}(\infty, 0)$ for some $j \in\{1,2, \ldots,(m-1) / 2\}$, then

$$
\sum_{n=0}^{\infty} n^{m-2 j-1} f\left(n, a(n-\delta)^{2 j}\right)<\infty
$$

for every $a>0$, and

$$
\sum_{n=0}^{\infty} n^{m-2 j} f\left(n, b(n-\delta)^{2 j-1}\right)=\infty
$$

for every $b>0$. Conversely, if (14) holds for every $b>0$ and (12) holds for some $C>0$, then equation (1) has an eventually positive solution in $O_{j}(\infty, 0)$.

Our final result is concerned with the existence of convergent positive solutions.

THEOREM 8. Suppose that $m$ is odd. Suppose further that $f$ is superlinear or sublinear. If equation (1) has an eventually positive solution which converges to a positive constant, then there is a constant $C>0$ such that

$$
\sum_{n=0}^{\infty} n^{m-1} f(n, C)<\infty
$$

The converse is also true.

Again, the proof is similar to that of Theorem 2. In particular, to show the converse, we first retain the definitions of the Banach space $B$ and its subspace $\Omega$ in the proof of Theorem 2. We then change the definition of $\Gamma(n)$ to $\Gamma(n)=1$ for $n \geq 0$. Furthermore, for the case $p \in[0,1)$, we modify the definition of $T$ so that

$$
(T x)_{n}=D \Gamma(M), \quad N \leq n<M,
$$


and

$$
(T x)_{n}=\frac{3 D(1+p)}{2}-p x_{n-\tau}+H_{n}^{m-1}(i) f\left(i, x_{i-\delta}\right), \quad n \geq M ;
$$

and for the case $p>1$, we modify the definition of $T$ so that

$$
(T x)_{n}=\frac{3 D(1+p)}{2 p}-\frac{1}{p} x_{M+\tau}, \quad N \leq n<M,
$$

and

$$
(T x)_{n}=\frac{3 D(1+p)}{2 p}-\frac{1}{p} x_{n-\tau}+\frac{1}{p} H_{n+\tau}^{m-1}(i) f\left(i, x_{i-\delta}\right), \quad n \geq M .
$$

The same reasoning as described in the proof of Theorem 2 leads to the conclusion that (1) has an eventually positive solution which converges to a positive constant.

As a final remark, when $m=4, p=0$ and $j=2$ in Theorem 2 , and when $m=4$, $p=0$ and $j=1$ in Theorem 3, the corresponding results have been derived by Zhang and Cheng [14, Theorem 3.1 and Theorem 3.2]. But in [14], only results related to fourth order equations are discussed.

\section{References}

[1] R. P. Agarwal, Difference equations and inequalities (Academic Press, New York, 1992).

[2] S. S. Cheng and W. T. Patula, 'An existence theorem for a nonlinear difference equation', Nonlinear Anal. 30 (1993), 193-203.

[3] S. S. Cheng, G. Zhang and W. T. Li, 'On a higher order neutral difference equation', Recent Trends in Math. Anal. Appl., to appear.

[4] S. R. Grace and B. S. Lalli, 'Oscillation theorems for second order delay and neutral difference equations', Util. Math. 45 (1994), 199-211.

[5] X. Z. He, 'Oscillation and asymptotic behavior of second order nonlinear difference equation', $J$. Math. Anal. Appl. 175 (1993), 482-498.

[6] I. T. Kiguradze and T. A. Chanturia, Asymptotic properties of solutions of nonautonomous ordinary differential equations (Kluwer, Amsterdam, 1993).

[7] B. S. Lalli, 'Oscillation theorems for neutral difference equations', Comput. Math. Appl. 28 (1994), 191-202.

[8] B. S. Lalli and B. G. Zhang, 'On existence of positive solution and bounded oscillation for neutral difference equations', J. Math. Anal. Appl. 166 (1992), 272-287.

[9] B. S. Lalli, B. G. Zhang and J. Z. Li, 'On the oscillation and existence of positive solutions of neutral difference equations', J. Math. Anal. Appl. 158 (1991), 213-233.

[10] W. T. Li and S. S. Cheng, 'Classifications and existence of positive solutions of second order nonlinear neutral difference equations', Funkcial. Ekvac. 40 (1997), 371-393.

[11] B. Liu and J. R. Yan, 'Oscillation theorems for nonlinear neutral difference equations', J. Differ. Equations Appl. 1 (1995), 307-315. 
[12] B. Smith and W. E. Taylor, Jr., 'Oscillatory and asymptotic behavior of certain fourth order difference equations', Rocky Mountain J. Math. 16 (1986), 403-406.

[13] A. Zafer and R. Dahiya, 'Oscillation of a neutral difference equation', Appl. Math. Lett. 6 (1993), 71-74.

[14] B. G. Zhang and S. S. Cheng, 'On a class of nonlinear difference equations', J. Differ. Equations Appl. 1 (1995), 391-411.

[15] G. Zhang and S. S. Cheng, 'Oscillation criteria for a neutral difference equation with delay', Appl. Math. Lett. 8 (1995), 13-17.

[16] X. L. Zhou and J. R. Yan, 'Oscillatory properties of higher order nonlinear difference equations', Comput. Math. Appl. 31 (1996), 61-68.

Institute of Mathematics

Gansu University of Technology

Lanzhou, Gansu 730050

P. R. China

Department of Mathematics

Datong Advanced College

Datong, Shanxi 037008

P. R. China

\section{Department of Mathematics}

Tsing Hua University

Hsinchu, Taiwan 30043

R. O.C. 